\title{
ESTUDO DE CFD SOBRE A INFLUENNCIA DA CONFIGURAÇÃO DA CHICANA EM TANQUES DE MISTURA
}

\author{
A. NOGUEIRA ${ }^{1}$, J.L GOMEZ ${ }^{2}$ e E. CEKINSKI ${ }^{1}$ \\ ${ }^{1}$ Instituto de Pesquisas Tecnológicas, IPT, Departamento de Processos Industriais. \\ ${ }^{2}$ Universidade Estadual de Campinas, Departamento de Engenharia Química. \\ E-mail para contato: andressapancioni@yahoo.com.br
}

\begin{abstract}
RESUMO - Atualmente o uso de ferramentas computacionais na simulação de tanques de mistura está sendo amplamente discutido, visto os benefícios com flexibilidade da proposta de soluções e redução de custo no projeto e otimização dos equipamentos. Assim, o objetivo deste trabalho é analisar a influência de diversos formatos de chicanas em um tanque agitado com um impelidor PBT, avaliando o número de potência, número de bombeamento, potência, tempo de mistura e o consumo de energia, bem como analisar o perfil do escoamento através de CFD. Os resultados demonstram que houve uma redução significativa do gasto de energia em uma das configurações propostas, chegando a uma economia de 25\%; assim como uma influência no padrão de escoamento do tanque. Este estudo ressalta a importância da chicana na otimização dos processos de agitação e mistura.
\end{abstract}

\section{INTRODUÇÃO}

O processo de mistura em tanques agitados pode ser encontrado em qualquer indústria, podendo ser a etapa mais importante de todo o processo produtivo. Portanto, sua eficiência tem um grande impacto no custo de produção e na qualidade do produto tanto de forma direta quanto em um processo intermediário. O regime de escoamento turbulento está presente na maioria dos processos de mistura e é responsável por promover o aumento das taxas de transferência de calor e massa e a chicana tem um papel fundamental para o sucesso desta etapa (Wilcox, 1998).

Embora a chicana seja um dispositivo que promove a mistura efetiva do sistema, especialmente neste tipo de regime, tem sido pouco estuda na literatura. Atualmente, há indícios de estudos relacionados a variações da chicana padrão, mas não há novas geometrias ou casos de estudo de tempo de mistura. Isso se deve ao fato de muitos estudos realizados não serem divulgados por gerarem segredo industrial. A instalação de chicanas destrói efetivamente o padrão circular do fluxo e inibe a formação de vórtice. Além disso, o fluxo axial se torna mais forte, melhorando a taxa de mistura. Por esses motivos, tanque com chicanas é o mais amplamente utilizado em aplicações industriais e tem recebido maior atenção dos experimentalistas e modeladores (McCabe et. al., 1985).

Deste modo, este trabalho pretende avaliar a influência de diferentes formatos de chicana em um tanque agitado em regime turbulento. Foi utilizando um impelidor pás inclinadas (PBT $45^{\circ}$ ), já que este impelidor é uns dos mais extensamente utilizados na 
indústria. Foram propostas três configurações de chicana (meia chicana, chicana afastada e chicana angular) e as mesmas foram comparadas ao formato padrão, sendo analisadas variáveis importantes nos processos de agitação e mistura, tais como número de potência e número de bombeamento, assim como as características dos fluxos. Na estimativa do consumo de energia foi utilizado um traçador numérico para calcular o tempo de mistura.

\section{MODELAGEM MATEMÁTICA}

O escoamento de um fluido pode ser descrito através de equações de transporte, mantendo como principio as leis de conservação de massa e quantidade de movimento. $\mathrm{Na}$ Equação 1 é representada a equação de conservação de massa, entretanto, as equações de conservação de quantidade de movimento nos três eixos coordenados (x,y,z), são representadas na forma inicial pela Equação 2 e são conhecidas como as equações de NavierStokes.

$$
\begin{aligned}
& \frac{\partial \rho}{\partial t}+\rho\left[\frac{\partial v_{i}}{\partial x_{i}}+\frac{\partial v_{j}}{\partial x_{j}}+\frac{\partial v_{k}}{\partial x_{k}}\right]=0 \\
& \rho\left[\frac{\partial v_{i}}{\partial t}+\frac{\partial\left(v_{i} \cdot v_{j}\right)}{\partial x_{j}}\right]=-\frac{\partial p}{\partial x_{i}}+\frac{\partial}{\partial x_{j}}\left[\mu\left(\frac{\partial v_{i}}{\partial x_{j}}+\frac{\partial v_{j}}{\partial x_{i}}-\frac{2}{3} \delta_{i j} \nabla \cdot \vec{v}\right)\right]+\rho g_{i}+\sum F_{i}
\end{aligned}
$$

Onde os termos; $\rho, \vec{v}, \mu, P, \vec{g}$ representam a densidade, velocidade, viscosidade, pressão e gravidade respectivamente. Entretanto o termo $F_{i}$, envolve as forças adicionais que aparecem em sistemas rotativo tais como a força centrifuga e de Coriolis (ANSYS, 2011).

Este sistema de equações, na maioria das vezes, não possui uma solução analítica geral, portanto é necessária uma discretização do domínio de estudo em volumes de controle para serem resolvidas numericamente.

\section{CONFIGURAÇÃO DO MODELO CFD}

Foram estudadas quatro geometrias de chicanas em um tanque com fundo toresférico de $10 \%$ e diâmetro $\mathrm{T}=2 \mathrm{~m}$. Foi utilizado um impelidor pás inclinadas (PBT $45^{\circ}$ ) de diâmetro T/3 para todos os casos. Na Figura 1 são mostradas configurações de geometrias das chicanas estudadas. 


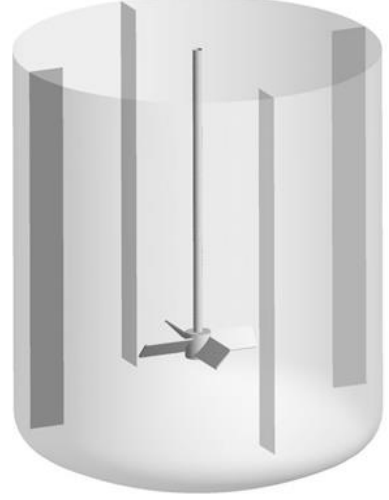

(a)

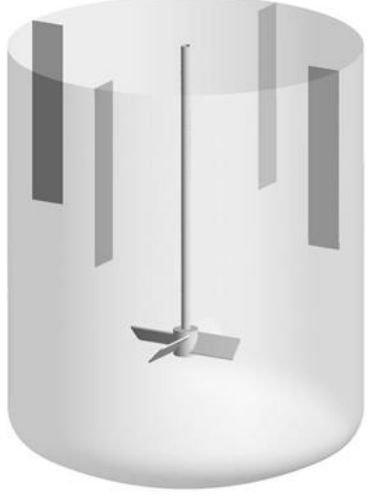

(b)

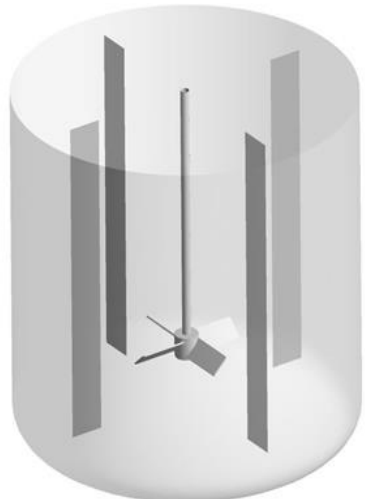

(c)

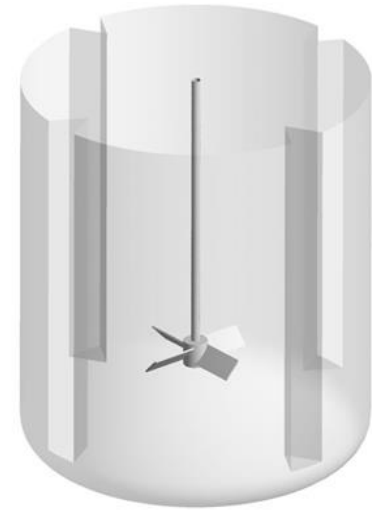

(d)

Figura 1 -Geometria das chicanas estudadas: a) chicana padrão, b) meia chicana, c) chicana afastada, d) chicana angular.

Foi utilizada uma malha 3D híbrida composta de elementos tetraédricos e prismáticos, já que esta possui as vantagens de se adaptar a geometrias complexas além de esquemas automáticos de criação da malha, sendo útil na análise de configuração e designs. A Figura 2 mostra a malha empregada em que foram realizados refinos locais nas superfícies e interfaces do tanque além da utilização de elementos prismáticos na parede do tanque e no impelidor. Foi realizado teste de refinamento da malha $\left(1,8 \times 10^{6} ; 1,4 \times 10^{6} ; 1 \times 10^{6}\right.$ elementos $)$ para análise do seu efeito no número de potência e número de bombeamento. Foi possível concluir que para a malha de $1,8 \times 10^{6}$ os resultados apresentam independência com variações menores do que $0,5 \%$, sendo inferior ao utilizado por outros autores (Brucato et al., 1998).

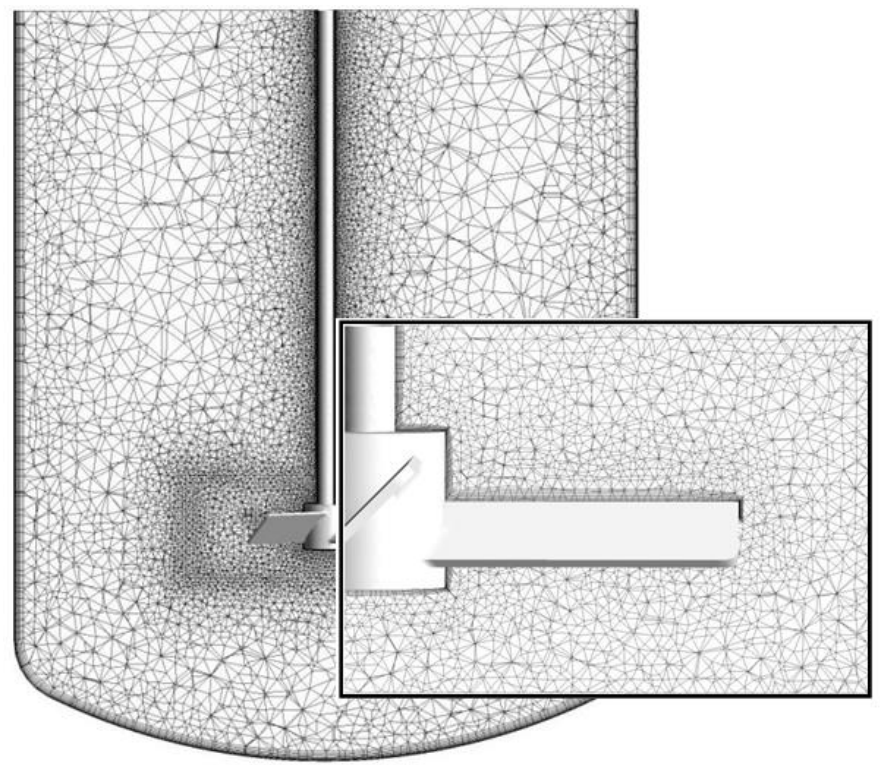

Figura 2 - Malha tetraédrica utilizada na discretização do domínio de estudo.

Todas as malhas foram geradas utilizando critérios estabelecidos no manual do usuário de ANSYS (2011). Entretanto, para um cálculo adequado das quantidades de transporte nas superfícies do tanque foi definido o refino da malha para garantir um y+ $<10$. A localização 
do domínio rotativo foi definida de acordo com sugestões feitas na literatura (Coroneo et al., 2011).

As simulações foram feitas no estado estacionário considerando um escoamento monofásico em regime turbulento $(\mathrm{Re}=50.000)$, sendo utilizando como fluido de trabalho a água. Para resolução numérica foi utilizado o pacote comercial Ansys CFX 14.0.

Esquemas numéricos de alta resolução bem como o modelo de turbulência Shear Stress Tranport (SST) desenvolvido por Menter (1994), foram utilizadas conforme sugestões de Nunhez et al. (2012) para esse tipo de sistema agitado. Condições de não escorregamento e superfície plana no topo do tanque foram empregadas nos contornos do domínio. Finalmente, uma abordagem de múltiplos sistemas de referência Frozen-Rotor foi utilizada para descrever o movimento do impelidor dentro do domínio rotativo.

\section{RESULTADOS E DISCUSSÕES}

Foi avaliado e comparado o efeito das diferentes configurações de chicana propostas com relação ao número de potência, número de bombeamento e padrão de fluxo do sistema de mistura através de CFD. Foi estimado o tempo de mistura mediante a adição de um traçador numérico. Um estudo de convergência foi realizado a fim de encontrar um critério de convergência adequado em que os erros de truncamento do método não influenciassem nos resultados numéricos. Portanto, foi escolhido um erro residual RMS (Root Mean Square) < $10^{-5}$, sendo verificado através da estabilidade do número de potência este parâmetro também usado por outros autores (Murthy e Joshi, 2008).

\subsection{Caracterização do Fluxo}

Pode-se observar uma direção de bombeamento descendente (pumping-down) para todos os casos, conforme mostrado na Figura 3. Isto está em concordância com a característica de fluxo axial deste tipo de impelidores. De acordo com o perfil de velocidade, nota-se que o fluxo gerado pelo impelidor no caso da chicana angular tem maior alcance radial, ou seja, o fluxo é impulsionado em direção à parede do tanque e sobe, descendo novamente após quase alcançar o topo do tanque. Esse movimento favorece a redução do tempo de mistura do sistema. As chicanas afastadas (c) apresentam um padrão mais circulatório, ou seja, há pouca circulação axial, e esta característica não contribui para a homogeneização da mistura. Com isso, demonstram também maior intensidade de mistura no fundo do tanque e pouca no topo do tanque. A chicana padrão e a meia chicana têm um fluxo mais intenso na região do eixo do impelidor do que as outras geometrias de chicana. 


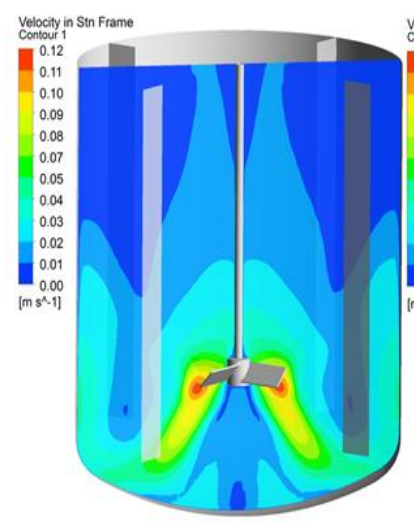

(a)

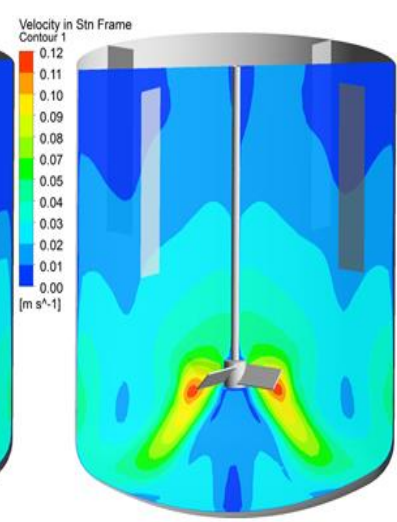

(b)

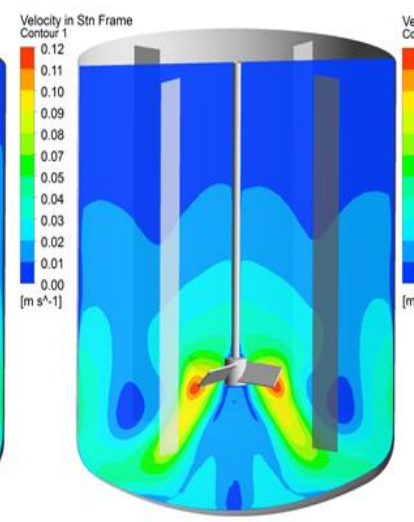

(c)

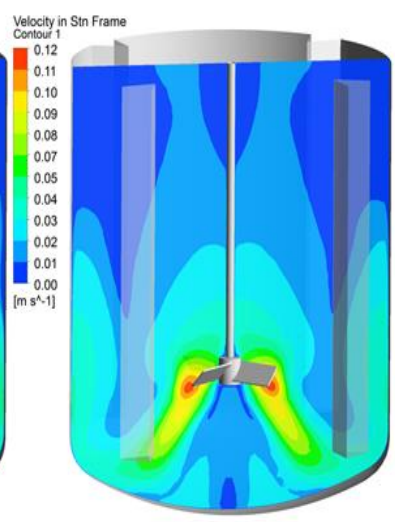

(d)

Figura 3 - Comparação do perfil de velocidade das chicanas estudadas: a) chicana padrão, b) meia chicana, c) chicana afastada, d) chicana angular.

Na Figura 4 são mostradas as linhas de corrente para os diversos formatos de chicana. Percebe-se que o formato (a) e (b) mostram um perfil de caminho semelhante, com mais intensidade perto do impelidor e também no eixo, porém, o fluido não passa em algumas partes do tanque. Já para o formato de chicana (c) mostra-se uma recirculação intensa no eixo do impelidor e no topo do tanque.

Essas características não ajudam reduzir o tempo de mistura. No entanto, na chicana (d) mostra um caminho mais caótico, ou seja, neste caso a partícula está se movimentando por todo o tanque e consequentemente promove uma mistura mais homogênea mais rapidamente.

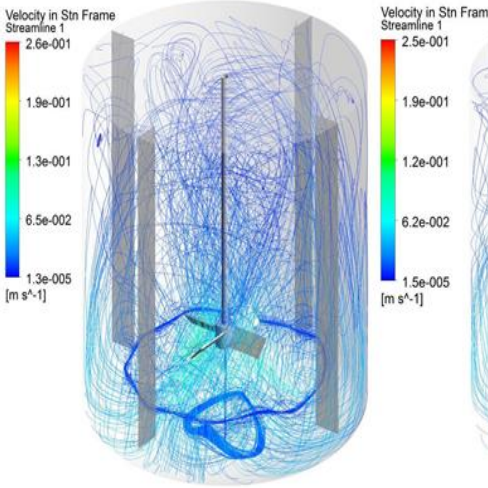

(a)

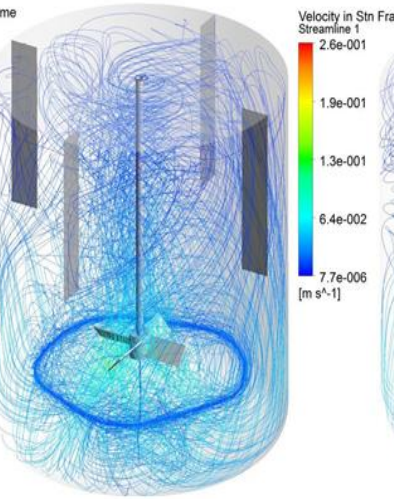

(b)

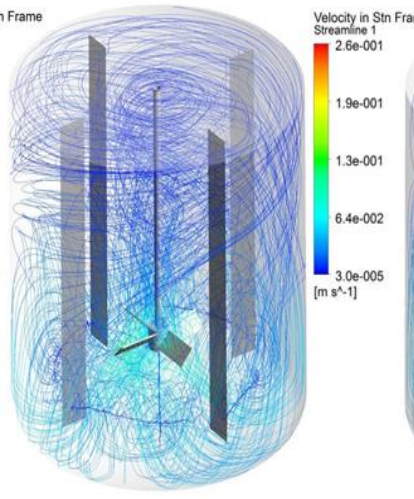

(c)

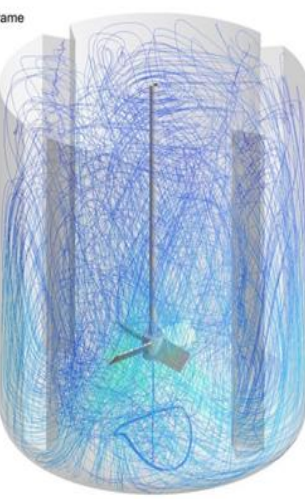

(d)

Figura 4 - Comparação das linhas de corrente para as chicanas estudadas: a) chicana padrão, b) meia chicana, c) chicana afastada, d) chicana angular.

\subsection{Tempo de Mistura}

O tempo de mistura para um tanque agitado pode ser definido como o tempo em que a mistura atinge um grau de uniformidade desejado. Para a estimativa do tempo de mistura é utilizado um traçador numérico, o qual é medido até alcançar uma concentração de equilíbrio. Segundo Cabrales et al. (2011), o tempo de mistura estimado é maior quando o traçador é iniciado no topo do tanque e, portanto, essa foi a posição adotada neste trabalho. 
Existem diversas metodologias empregadas para o cálculo do tempo de mistura, no entanto autores como Streiff (1979), sugerem que o coeficiente de variação (CV) é o parâmetro mais representativo para estimar o tempo de mistura. Foi considerado como critério de homogeneização para este trabalho $\mathrm{CV}=0,01$, o qual corresponde ao tempo no qual as variações da concentração de traçador são da ordem de $1 \%$. A Equação 3 descreve o cálculo da variação.

$$
\sigma^{2}=\frac{1}{N-1} \sum_{i=0}^{i=p}(C i-\bar{C})^{2}
$$

Na figura 5 se observa que para as chicanas afastadas, o traçador fica perto da região do impelidor e ainda contempla uma grande quantidade no topo do tanque. Já para a chicana angular o traçador já está praticamente em todo o volume do tanque. A chicana padrão e a meia chicana têm melhor distribuição do que as chicanas afastadas, porém ainda com vários pontos sem traçador.

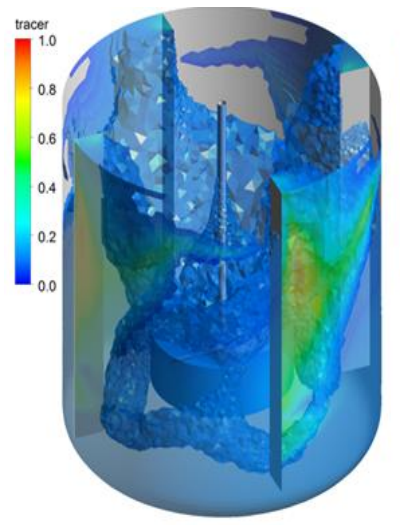

(a)

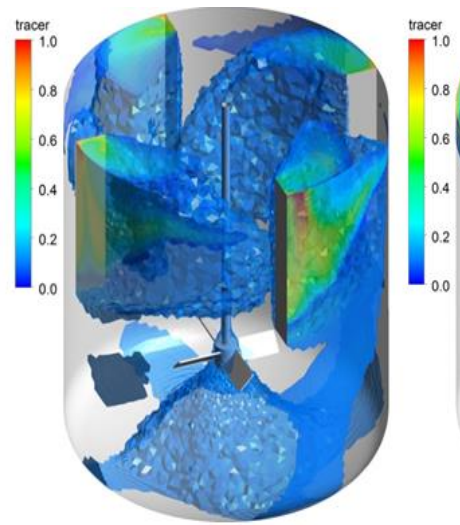

(b)

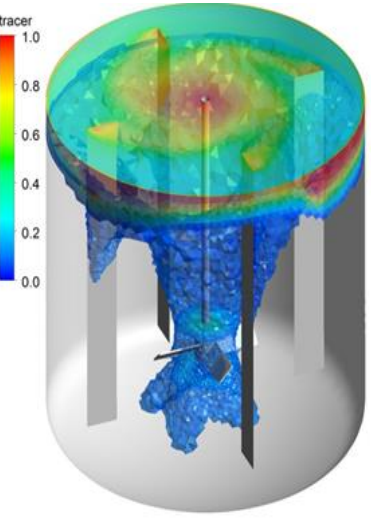

(c)

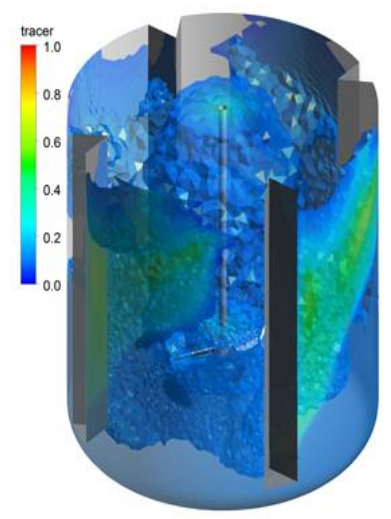

(d)

Figura 5 - Comportamento da concentração do traçador após de $\mathrm{t}=240 \mathrm{~s}$ de simulação para as configurações de chicana: a) chicana padrão; b) meia chicana. c) chicana afastada. d) chicana angular.

\subsection{Análise de Variáveis}

A Tabela 1 mostra os dados de número de potência $(\mathrm{Np})$, número de bombeamento $(\mathrm{Nq})$, tempo de mistura, potência consumida e gasto de energia para a configuração de chicana padrão, meia chicana, chicana afastada e chicana com ângulo. A estimativa destas variáveis foi realizada utilizando funções definidas pelo usuário e através de sub-rotinas estabelecidas no software ANSYS-CFX. 
Tabela 1 - Estimativa de variáveis de análise para todas as configurações de chicana

\begin{tabular}{|c|c|c|c|c|c|}
\hline Tipo de Chicana & $\mathrm{Np}$ & $\mathrm{Nq}$ & Tempo de mistura [s] & Potência [W] & Energia [W] \\
\hline Padrão & 1,12 & 0,67 & 570 & 0,21 & 119,78 \\
Meia & 1,02 & 0,66 & 648 & 0,19 & 123,65 \\
Afastada & 1,1 & 0,67 & 1266 & 0,21 & 261,03 \\
Angular & 1,1 & 0,66 & 438 & 0,21 & 90,80 \\
\hline
\end{tabular}

Comparando-se com a chicana padrão, há uma redução em torno de $9 \%$ na potência consumida pela configuração de meia chicana, mas este parâmetro fica praticamente constante para as outras geometrias. Porém esse parâmetro, se analisado isoladamente, pode levar a uma conclusão equivocada, uma vez que, nestes casos, deve também ser analisado em conjunto do tempo de mistura.

Não há variação significativa para o número de bombeamento, portanto o mesmo fica estável para todas as configurações. Esse resultado está dentro do esperado uma vez que esta variável está relacionada com o tipo de impelidor.

O tempo de mistura aumentou em $13 \%$ para a meia chicana e em $122 \%$ para a chicana afastada em relação à chicana padrão. O perfil de fluxo gerado pelas geometrias influencia consideravelmente no tempo de mistura. Para o caso da chicana afastada, há muitos pontos de zona morta no tanque bem como uma circulação giratória em torno do eixo que desfavorece a mistura efetiva do fluido. Já a meia chicana apresenta rotação sólida no fundo e na metade do tanque, esse comportamento compromete o tempo de mistura. A configuração de chicana angular foi a única que reduziu o tempo de mistura e a porcentagem atingida foi de $23 \%$; isso se deve ao fato dessa geometria ter gerado um perfil caótico do fluxo, promovendo a mistura efetiva, evitando também a rotação sólida no tanque de mistura.

A configuração de chicana com ângulo foi a que apresentou menor gasto com energia entre todas as configurações, bem como menor tempo de mistura. Comparando a redução do gasto, chega a uma redução de $24 \%$ em relação à chicana padrão e a $65 \%$ com relação à chicana afastada, que é uma configuração comumente utilizada na indústria para evitar encrustamento.

Esse ganho é muito representativo para a indústria, uma vez que consume menos energia e reduz o tempo de operação do equipamento, o que pode ser traduzido em um aumento de capacidade e redução de custos na produção, tornando a compra de novos equipamentos desnecessária.

\section{CONCLUSÃO}

Através da análise CFD pode-se concluir que a chicana com ângulo reduz consideravelmente o tempo de mistura e a potência consumida com relação à chicana padrão. Conforme os resultados apresentados, esse sistema apresentou o fluxo mais caótico, promovendo uma mistura efetiva. Já a chicana afastada criou várias zonas mortas, dobrando o consumo de potência e tempo de mistura. Por outro lado, a meia chicana teve um desempenho parecido com a chicana padrão. 
É importante salientar que a simulação vem se tornando uma ferramenta poderosa para o conhecimento de tanques agitados, uma vez que pode prever problemas de mistura, aperfeiçoar a qualidade dos produtos e reduzir tempos de mistura através de estudos de novos casos. Este trabalho ressalta a importância da chicana para sistemas de mistura em regime turbulento e também documenta um estudo de geometrias de chicana, uma vez que há poucos trabalhos com este tema.

\section{BIBLIOGRAFIA}

ANSYS, Inc. Solver Theory Guide. ANSYS CFX 14.0, 2011.

BRUCATO, A.; CIOFALO, M.; GRISAFI, F.; MICAELE, G. Numerical prediction of flow fields in baffled stirred vessels: A comparison of alternative modelling approaches. Chem. Eng. Science, v. 53, 3653-3684, 1998.

CABRALES, F. A. N.; SPOGIS, N.; NUNHEZ, J. R.; MARTIGNONI, W. P.; CASTRO, H. C. A.; ITO, A. S. Computational fluid dynamic study of mixing in large scale diesel storage vessels. AIChE Spring Meeting \& 7th Global Congress on Process Safety, Chicago, v. a. p. 211173-1-211173-9, 2011.

CORONEO, M.; MONTANTE, G.; PAGLIANTI, A.; MAGELLI, F. CFD prediction of fluid flow and mixing in stirred tanks: Numerical issues about the RANS simulations. Comp. \& Chem. Eng., 35, 1959-1968, 2011.

McCABE, W. L.; SMITH, J. C.; HARRIOTT, P. Unit Operations of Chemical, ed. $4^{\text {th }}$, McGraw-Hill, 1985

MENTER, F. R. Two-equation eddy-viscosity turbulence models for engineering applications. AIAA-Journal, 32, 1598 - 1605, 1994.

MURTHY, B. N.; JOSHI, J. B. Assessment of standard, RSM and LES turbulence models in a baffled stirred vessel agitated by various impeller designs. Chem. Eng. Science, 63, 5468-5495, 2008.

NUNHEZ, J. R.; KRESTA, S. M.; MACHADO, M. B.; GOMEZ, J.L. Investigating on the influence of mesh density, turbulence models and numerical aspects of CFD modeling in PBT Impellers using non-structured meshes. AIChE Annual Meeting, Pittsburgh, 526e, 282309, 2012.

STREIFF, F. A., Adapted motionless mixer design, in "Proc. Third European Conference on Mixing", 1979, pp. 171-188.

WILCOX, D. C. Turbulence modeling for CFD. 2 ed. USA: DCW Industries, 1998. 